\title{
Optimising utilisation of kidneys from very young deceased donors: the technique of en bloc kidney transplantation
}

Ziting Wang ${ }^{1}$, MBBS, MRCS, Hui Kim $\underline{\text { Yap }}^{2}$, MD, FRCPCH, Krishnan Prabhakaran ${ }^{3}$, FRCSG, FAMS, Ho Yee $\underline{\text { Tiong }}{ }^{1}$, MMed, FAMS

ABSTRACT Kidneys of paediatric deceased donors were previously considered suboptimal for older recipients. An 18-month-old deceased donor was made available via Singapore's Medical (Therapy, Education and Research) Act. To the best of our knowledge, she is the youngest local donor. We herein report a case of successful kidney transplantation, using the en bloc technique, to a 15-year-old girl with renal failure secondary to bilateral cystic dysplastic kidney.

Keywords: cadaveric kidneys, en bloc, paediatric donors, renal transplant, Singapore

\section{INTRODUCTION}

We herein report successful kidney transplantation using the en bloc technique. The deceased donor was 18 months old, which made her the youngest local donor in our knowledge, and was available through Singapore's Medical (Therapy, Education and Research) Act.

\section{CASE REPORT}

The recipient was a 15-year-old girl with renal failure secondary to bilateral cystic dysplastic kidney. During procurement, the donor's kidneys were removed, with the aorta and vena cava (VC) attached and retaining the surrounding adventitia (Fig. 1). The proximal end included the superior mesenteric arterial branch, while the distal end contained the junction of the aorta and common iliac arteries. It is essential to ensure that the adventitial tissue is of adequate length.

Backbench preparation of the en bloc kidneys involved careful ligation of lumbar vessels and the removal of excess perirenal fat. The upper borders of the donor aorta and VC were oversewn to reduce the number of anastomoses, hence decreasing the likelihood of thromboses. During surgery, the kidneys were placed extraperitoneally in the right iliac fossa medial and lateral to each other, and fixed to the lateral pelvic wall to reduce tension on the vasculature. ${ }^{(1)}$ The reconstructed donor VC was anastomosed to the external iliac vein and donor aorta to the common iliac artery (end-to-side). The two donor ureters were anastomosed to the bladder over two 4.7 Fr double-J stents with an anti-reflux technique.

Total operative time, including bench work, was 192 minutes, with a cold ischaemia time of 480 minutes, warm ischaemia time of one minute and anastomotic time of 40 minutes. There was immediate graft function and creatinine levels remained stable at $45 \mu \mathrm{mol} / \mathrm{L}$ at one year. The two kidneys demonstrated hypertrophy, each increasing to $9.5 \mathrm{~cm}$ in length after one year from $7.6 \mathrm{~cm}$ and $7.8 \mathrm{~cm}$.

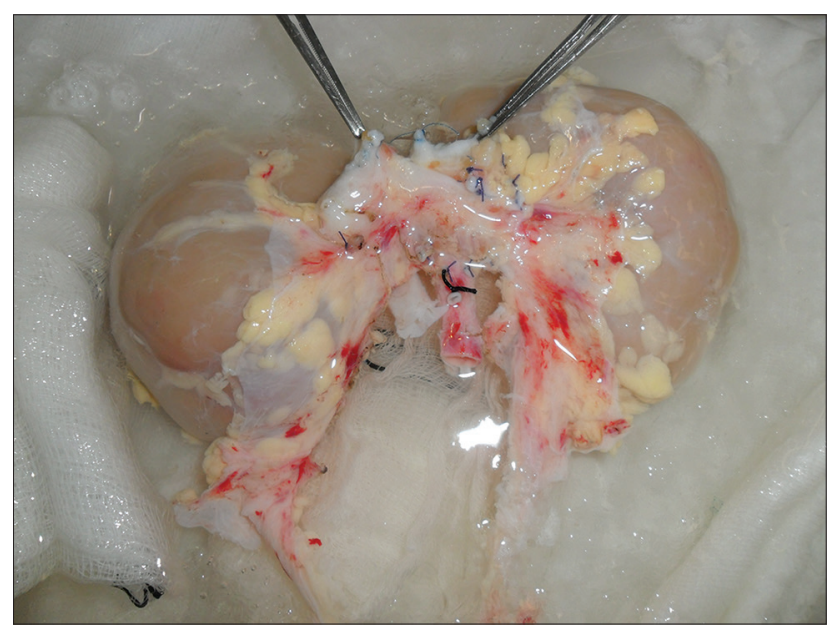

Fig. 1 Photograph shows anterior view of en bloc procurement of the patient's kidney with preservation of the aorta and vena cava length. Minimal dissection was employed at the hilum to prevent injury to small vessels and ureters.

\section{DISCUSSION}

The number of chronic renal failure patients undergoing dialysis has increased from 3,565 in 2005 to 4,378 in 2009, whilst the number of renal transplants remained in the hundreds. ${ }^{(2)}$ The adult deceased donor is a key source for transplants. However, there is an inadequate pool of paediatric donors. The widening disparity may be addressed by recourse to non-conventional donors.

Although medical concerns remain about reduced functional reserves and hyperfiltration, graft survival in cases where donors are aged 0-5 can be improved through better matching of donor-recipient weights. ${ }^{(3)}$ Our case report demonstrates that previously feared surgical complications such as graft thrombosis and ureteric complications can be overcome with the en bloc kidney transplantation technique. We hope to highlight the option of the paediatric deceased donor to supplement kidney transplants from deceased donors through the Human Organ Transplant Act.

${ }^{1}$ Department of Urology, ${ }^{2}$ Department of Paediatrics Renal Medicine, ${ }^{3}$ Department of Paediatric Surgery, National University Health System, Singapore

Correspondence: Dr Ho Yee Tiong, Senior Consultant, Department of Urology, National University Hospital, 5 Lower Kent Ridge Rd, Singapore 119074. cfsthy@nus.edu.sg 


\section{REFERENCES}

1. Ekser B, Baldan N, Margani G, et al. Monolateral placement of both kidneys in dual kidney transplantation: low surgical complication rate and short operating time. Transpl Int 2006; 19:485-91.

2. Choong HL, ed. Eighth Report of the Singapore Renal Registry 2009.
Singapore: Health Promotion Board, 2012.

3. Bresnahan BA, McBride MA, Cherikh WS, Hariharan S. Risk factors for renal allograft survival from pediatric cadaver donors: an analysis of united network for organ sharing data. Transplantation 2001; 72:256-61. 